\section{CHILD CAR RESTRAINT USE AMONG ABORIGINAL AND TORRES STRAIT ISLANDER CHILDREN}

1,2,3 Kate Hunter, ${ }^{1,2}$ Lisa Keay, ${ }^{4}$ Kathleen Clapham, ${ }^{5}$ Julie Brown, ${ }^{5}$ Lynne E Bilston, 1,2 Sharmane Mannix, 1,2 Jake Byrne, ${ }^{2}$ Judy M Simpson, ${ }^{1,2}$ Rebecca Q Ivers. ${ }^{1}$ The George Institute for Global Health, Australia; ${ }^{2}$ The University of Sydney, Australia; ${ }^{3}$ The Poche Centre for Indigenous Health, Australia; ${ }^{4}$ Australian Health Services Research Institute, University of Wollongong, Australia; ${ }^{5}$ Neuroscience Research Australia, the University of New South Wales, Australia

\subsection{6/injuryprev-2016-042156.58}

Background In Australia, road related fatality rates for Aboriginal and Torres Strait Islander children aged $0-4$ years are 4 times higher than for other Australian children the same age. Children are less likely to be severely injured in a car crash if they are restrained in an age-appropriate car restraint and if the restraint is used correctly. Despite this, little is known about how Aboriginal children are travelling and whether or not they are being correctly restrained in age appropriate child restraints.

Methods Working with community and following extensive consultation and engagement in four urban communities in New South Wales, Australia, we recruited and trained local Aboriginal people to conduct surveys with parents and carers and to observe how children were restrained as they arrived at early childhood services attended by community members.

Results In 2015, we conducted interviews with 147 parents and carers and completed 109 observations of child restraint use at the 4 study sites. Parents or carers provided responses to the structured survey for 183 children. The average age was $3.0 \pm 1.3$ years (range $0-7$ years) and 137/183 (75\%) were Aboriginal or Torres Strait Islander children. There were 36/176 $(20 \%)$ not in the right restraint for their age; significant errors ranged from belt buckle not being engaged (11\%) to internal/ shoulder harness being incorrectly or not used (31\%).

Conclusions These findings are the first stage of the baseline data collection for a large trial involving 12 Aboriginal communities across New South Wales. It is the first large scale trial to measure the effectiveness of a culturally appropriate child restraint program among Aboriginal people in Australia. In a country where adult restraint use is close to $100 \%$, these preliminary findings highlight the need for a program aimed at increasing the proportion of Aboriginal and Torres Strait Islander children restrained in age appropriate restraints.

\section{CAPACITY BUILDING IN INDIGENOUS COMMUNITIES THRU COOPERATIVE AGREEMENTS}

${ }^{1}$ Nancy M Bill, ${ }^{2}$ Monique Sheppard. 'Indian Health Service, US Public Health Service, Rockville, Maryland, USA; ${ }^{2}$ Econometrica, Inc., Bethesda, Maryland, USA

\subsection{6/injuryprev-2016-042156.59}

Background The indigenous tribal groups of North American (American Indian and Alaska Native, AI/AN) suffer from higher rates of fatal unintentional injuries, specifically motor vehicle related injuries, in comparison to all US population. The Indian Health Service (IHS) is the US Federal Agency responsible for providing health services to members of federally-recognised AI/ AN by a government to government relationship established by law. IHS health and preventive services include injury prevention (IP). In 1997, IHS initiated national funding thru a cooperative agreement grant (CA) process aimed at building tribal capacity and infrastructure in injury prevention. CA grants have substantial involvement by awarding agency and the grant recipient. CA involves collaboration, participation or intervention in the program activities. An external monitoring contractor is hired for technical assistance to the grantees.

Methods The five-year CA provided funding to hire and train fulltime staff. Strategies targeted motor vehicle injury prevention through policy development, increasing occupant restraints, engaging key stakeholders, and roadway hazard identification. In addition, occupant restraint observational surveys, employing effective strategies, and on-going evaluation occurred in the five years.

Results The AI/AN grantee took the lead in the planning, organising, implementing and evaluating their programs to address motor vehicle related injuries and fatalities. Results included increasing adult seat belt use by 75 per cent and distributing 8,900 child safety seats, costing approximately $\$ 508,155$ and generating over $\$ 21.3$ million in benefits to society.

Conclusions IP's mission seeks to build the capacity of the US indigenous tribes to increase understanding of the injuries; to implement effective strategies to address the injury disparities. Utilising CA grants had great successes attributing to injury reduction in vulnerable populations. Many successes have been achieved over the years in decreasing the high motor vehicle injury fatality rates.

\section{YARNING, GIVING A VOICE TO OLDER ABORIGINAL PEOPLE ON HEALTHY AGEING AND FALL PREVENTION}

${ }^{1,2}$ J Coombes, ${ }^{1} \mathrm{C}$ Lukaszyk, ${ }^{1} \mathrm{~L}$ Keay, ${ }^{1} \mathrm{C}$ Sherrington, ${ }^{1} \mathrm{~A}$ Tiedemann, ${ }^{1,3} \mathrm{R}$ Ivers. ${ }^{1}$ The George Institute for Global Health, University of Sydney Australia; ${ }^{2}$ Lowitja Institute; ${ }^{3}$ Flinders University

\subsection{6/injuryprev-2016-042156.60}

Background There is emerging evidence that falls are an increasing problem for older Aboriginal people in Australia. We examined concepts of healthy ageing and fall prevention using Aboriginal ontology and knowledge systems through yarning circles in Aboriginal communities in Australia.

Methods We used a conversational method to gather knowledge through yarning circles with Aboriginal communities in the Central Coast, Dubbo, Mt Druitt and Shoalhaven areas of New South Wales, Australia. The yarning circles were held with 80 people aged from 45 to 85 years of age, in 8 groups between November 2014 and April 2015. Data were audio recorded and transcribed with the consent of communities and analysed using an Indigenous research standpoint methodology, incorporating ways of knowing, doing and being.

Results Yarning circles helped identify key issues around healthy ageing including the role of falls, in particular the impact these have on individuals, their families and communities. Discussions around falls highlighted concerns that they would lead to an inability to fulfil family roles or remain involved in community life. Participants reported that healthy ageing and maintaining independence were imperative in enabling them to continue to pass on cultural knowledge, and that they were comfortable attending health-related programs in their own communities.

Conclusions Aboriginal people felt comfortable attending programs in their own community and yarning circle participants voiced strongly that healthy ageing is essential for them to continue to share their knowledge of Aboriginal history and culture to their families and communities. Yarning circles also identified the need for Aboriginal-specific, culturally appropriate fall prevention programs to address healthy ageing and concerns about falls. 